\section{Self-Assembled Columns of Stacked Lipid Bilayers Mediated by Metal Ion Recognition}

Tina A. Waggoner ${ }^{\dagger}$, Julie A. Last ${ }^{\dagger}$, Paul G. Kotula ${ }^{\ddagger}$, and Darryl Y. Sasaki ${ }^{\dagger}$.

'Biomolecular Materials and National Laboratories

${ }^{\ddagger}$ Materials Characterization Dept., MS 1405

Albuquerque, NM 87185.

Received Date (will be automatically inserted after manuscript is accepted).

Molecular self-assembly can generate elaborate two- and three-dimensional supramolecular structures through the complementary interaction of attractive and repulsive forces between molecular components and their environment. ${ }^{\prime}$ Synthetic self-assembling systems are currently limited to the one-step creation of supramolecular structures from molecular components, unlike biological systems which can organize supramolecular assemblies into higher ordered structures to create tissue and functional materials of specific dimensions. ${ }^{2}$ Herein, we report the first observation of a synthetically produced higher ordered self-assembled structure of lipid bilayers with self-limiting dimensions mediated by chemical recognition at the membrane surface. Transmission electron microscopic (TEM) images of the self-assembled material reveal a columnar structure of stacked lipid bilayers uniform in width with lengths spanning dozens of bilayer thickness.

Previous work found that liposomes composed of a pyrenelabeled synthetic receptor lipid mixed into a distearylphosphatidylcholine (DSPC) matrix performed as highly selective optical sensors for heavy metal ions. ${ }^{345}$ In the absence of metal ions, the liquid-phase receptor lipid separates from the solid-phase DSPC matrix, producing fluorescence spectra with large excimer emission $\left(\lambda_{\max }=470\right.$ $\mathrm{nm})$ and relatively small monomer emission $\left(\lambda_{\max }=375 \mathrm{~nm}\right)$. Addition of di- or trivalent metal ions causes an inversion of the fluorescence emission peaks (excimer emission attenuates as the monomer emission intensifies), revealing dispersion of the receptor lipids into the DSPC matrix upon the metal ion recognition. Complete reversibility of the process was possible by removal of the metal ions with EDTA.
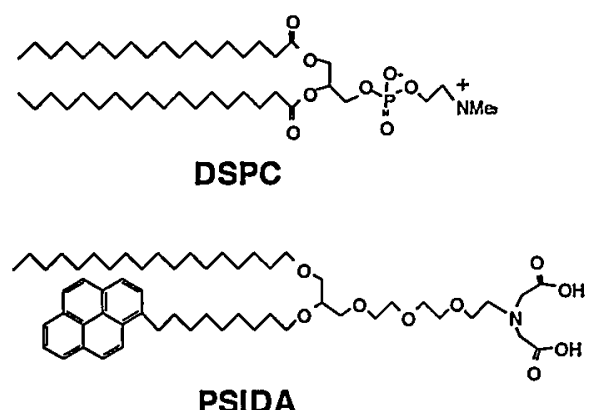

Figure 1. Matrix lipid DSPC and pyrene-labeled lipid PSIDA.

(1) (a) Lehn, J.-M. Science 1993, 260, 1762. (b) Whitesides, G. M.; Mathias, J. P.; Seto, C. T. Science 1991, 254, 1312.

(2) Alberts, B.; Bray, D.; Lewis, J.; Raff, M.; Roberts, K.; Watson, J. D. Molecular Biology of The Cell; third ed.; Garland Publishing: New York, 1994

(3) Sasaki, D. Y.; Shnek, D. R.; Pack, D. W.; Arnold, F. H. Angew. Chem., Int. Ed. Engl. 1995, 34, 905.

(4) Sasaki, D. Y.; Padilla, B. E. Chem: Comm. 1998, 1581.

(5) Sasaki, D. Y.; Waggoner, T. A. Proc. SPIE-Int. Soc. Opt. Eng. $1999,3606,46$.
Addition of $\mathrm{Cu}^{2+}$ to lipid bilayers composed of $5 \%$ PSIDA/DSPC (Figure 1) at $0.1 \mathrm{mM}$ lipid concentration caused the solution to become turbid, indicative of vesicle aggregation. The turbidity was, like the metal ion response, reversible upon the addition of EDTA. Turbid solutions were not observed with other receptor lipids functionalized with dithioamide or a crown ether that were selective for $\mathrm{Hg}^{2+}$ or $\mathrm{Pb}^{2+}$, respectively, nor with the 5\% PSIDA/DSPC liposomes in the presence of other divalent metal ions, such as $\mathrm{Mn}^{2+}$ or $\mathrm{Ca}^{2+}$.

In an effort to characterize the aggregate structures formed upon metal ion recognition, the bilayers were imaged by electron microscopy before and after addition of $\mathrm{Cu}^{2+}$. The liposomes were prepared as described previously ${ }^{5}$ and negatively stained using a standard TEM preparation protocol. 6 TEM images were taken on a Philips CM-30 operated at $300 \mathrm{kV}$. Figure 2 is an image of small unilamellar vesicles (SUV) of 5\% PSIDA/DSPC bilayers formed in MOPS buffered water $(0.02 \mathrm{M}$ MOPS, $0.10 \mathrm{M} \mathrm{NaCl})$ at $\mathrm{pH} 7.4$. Two free-floating liposome structures are observed in the middle of the image. Liposome sizes ranged between $400-700 \mathrm{~nm}$ in diameter. In addition, various forms of unstructured bilayer aggregates can be seen on the top and bottom of the image, accounting for approximately $50 \%$ of the observed features.

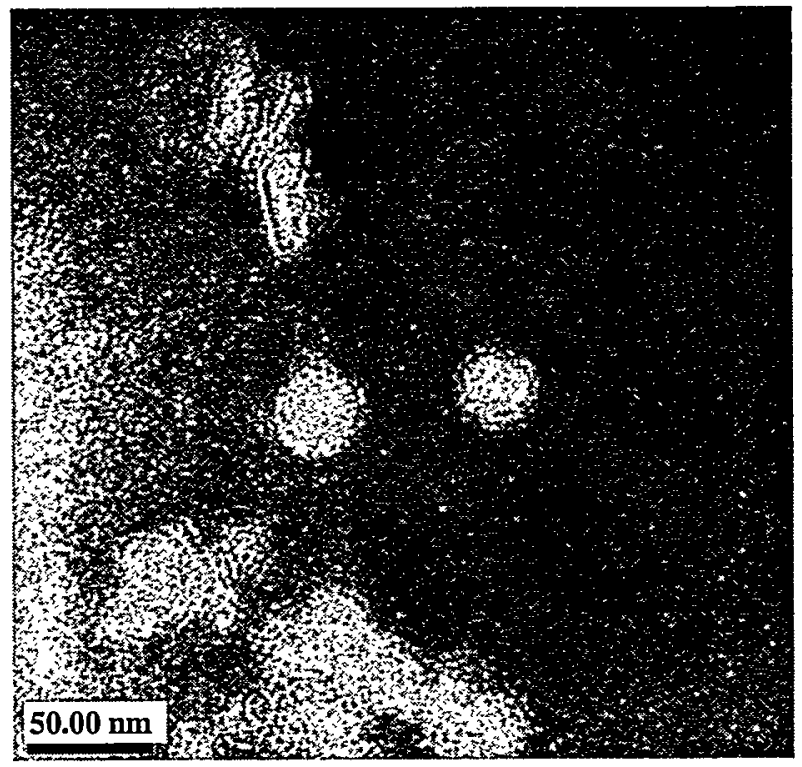

Figure 2. TEM image of 5\% PSIDADSPC bilayers in MOPS buffered water at $\mathrm{pH} 7.4$ (ammonium molybdate stain).

Upon addition of $\mathrm{Cu}^{2+}(1.0 \mu \mathrm{M})$ to this solution a remarkable self-assembly of the bilayers into columnar structures results. A representative image is shown in Figure 3, with more images available in the Supporting Information. Approximately 15 $20 \%$ of the observed bilayer structures on the TEM grid were in the form of these columns, the rest as aggregates and free liposomes. These unique structures have been reproducibly prepared.

The bilayer stacks exhibit uniform width throughout most of the structure. Other self-organizing lipid bilayer structures formed through bilayer-DNA ${ }^{7}$ or bilayer-actin ${ }^{8}$ complexation,

(6) New, R. R. C. Liposomes; Oxford University Press: New York, 1990.

(7) (a) Rädler, J. O.; Koltover, I.; Salditt, T.; Safinya, C. R. Science 1997, 275, 810. (b)Artzner, F.; Zantl, R.; Rapp, G.; Rädler, J. O. Phys. Rev. Lett. 1998, 81, 5015. 


\section{DISCLAIMER}

This report was prepared as an account of work sponsored by an agency of the United States Government. Neither the United States Government nor any agency thereof, nor any of their employees, make any warranty, express or implied, or assumes any legal liability or responsibility for the accuracy, completeness, or usefulness of any information, apparatus, product, or process disclosed, or represents that its use would not infringe privately owned rights. Reference herein to any specific commercial product, process, or service by trade name, trademark, manufacturer, or otherwise does not necessarily constitute or imply its endorsement, recommendation, or favoring by the United States Government or any agency thereof. The views and opinions of authors expressed herein do not necessarily state or reflect those of the United States Government or any agency thereof. 


\section{DISCLAIMER}

Portions of this document may be illegible in electronic image products. Images are produced from the best available original document. 
metal ion coordination, or biotin-streptavidin molecular recognition ${ }^{10}$ do not exhibit such self-limiting dimensions. These columnar structures varied in width from 600 to $900 \AA$ with lengths between several $(300 \AA)$ to $\sim 45(3300 \AA)$ bilayer thickness. The structure appears to be composed of individual lipid bilayers $\sim 40 \AA$ thick with $\sim 30 \AA$ spacing between each layer. It does not appear that these are flattened liposomes for the edges of each bilayer in the stack are discreet with no connectivity with its adjacent neighbor. One end of each stack there is a short, poorly organized stack of bilayers that is approximately half the diameter of the column. At the other end the stacks tend to taper off in size in the final few bilayers, sometimes leading off to another poorly organized stack.

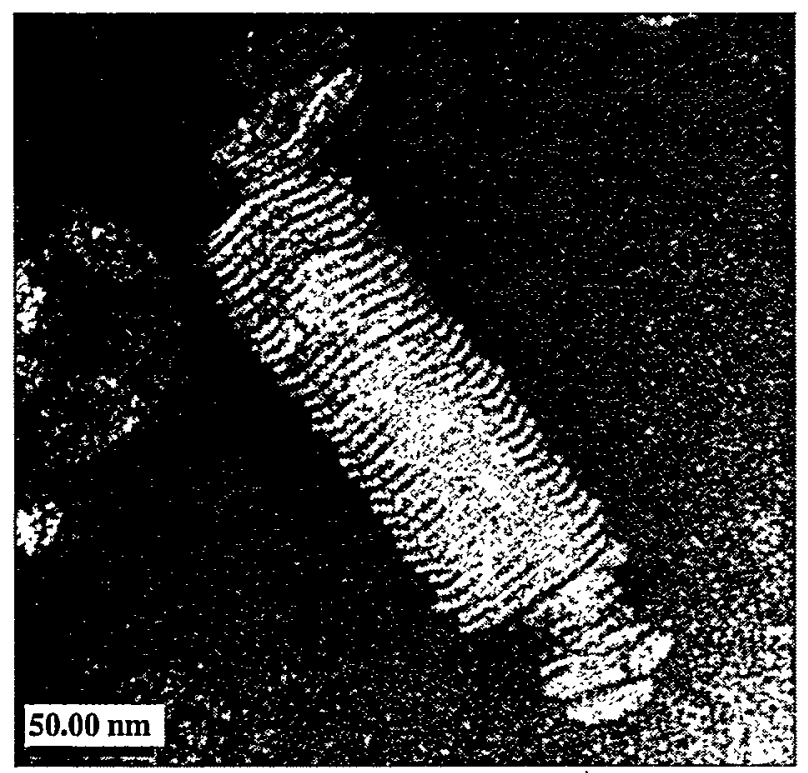

Figure 3. TEM image of self-assembled, columnar structured lipid bilayer stack that forms after $\mathrm{CuCl}_{2}(1.0 \mu \mathrm{M})$ addition to liposomes of 5\% PSIDA/DSPC.

The mechanism of formation of these self-assembled structures with uniform width is difficult to imagine considering that the initial liposome sizes are polydisperse. From the fluorescence data, we do know that $\mathrm{Cu}^{2+}$ binding to the iminodiacetic acid (IDA) receptor induces a dispersion of PSIDA molecules into the lipid matrix. Coordination of $\mathrm{Cu}^{2+}$ to the IDA headgroup leaves open an equivalent coordination site for another IDA to bind. The fluorescence data indicates that intra-liposome 2:1 coordination of IDA to $\mathrm{Cu}^{2+}$ does not occur, most probably due to geometric constraints of the twodimensional membrane surface. Orientation of the $\mathrm{Cu}^{2+}-\mathrm{IDA}$ complex perpendicular to the surface would, however, allow complexation with an IDA from another liposome. Through this metal ion-coordination-mediated event, numerous adhesion points between the two liposomes would rapidly propagate resulting in a flattening of the opposing bilayers, as illustrated in Figure 4. The dispersion of receptors due to metal ion binding should aid in creating a uniform adhesion between the bilayer surfaces. At some point, the liposome ruptures as a result of deformation from the adhesion process, allowing $\mathrm{Cu}^{2+}$

(8) Wong, G. C. L.; Tang, J. X.; Lin, A.; Li, Y.; Janmey, P. A.; Safinya, C. R. Science 2000, 288, 2035.

(9) Constable, E. C.; Meier, W.; Nardin, C.; Mundwiler, S. Chem. Comm. 1999, 1483.

(10) Chiruvolu, S.; Walker, S.; Israelachvili, J.; Schmitt, F.-J.; Leckband, D.; Zasadzinski, J. A. Science 1994, 264, 1753. to reach receptors in the liposome's interior. Although we have no confirming images at this time, the bilayers are most likely in the shape of a disc, as one might think of a flattened sphere. The first one or two bilayers formed may serve as a template for successive stacking of bilayers, regulating the width through the adhesion points between bilayers. The selforganizing nature of lipid assemblies may allow the bilayers to restructure, either recruiting or displacing lipids from adjacent bilayers to form a contiguous bilayer that matches the template size.

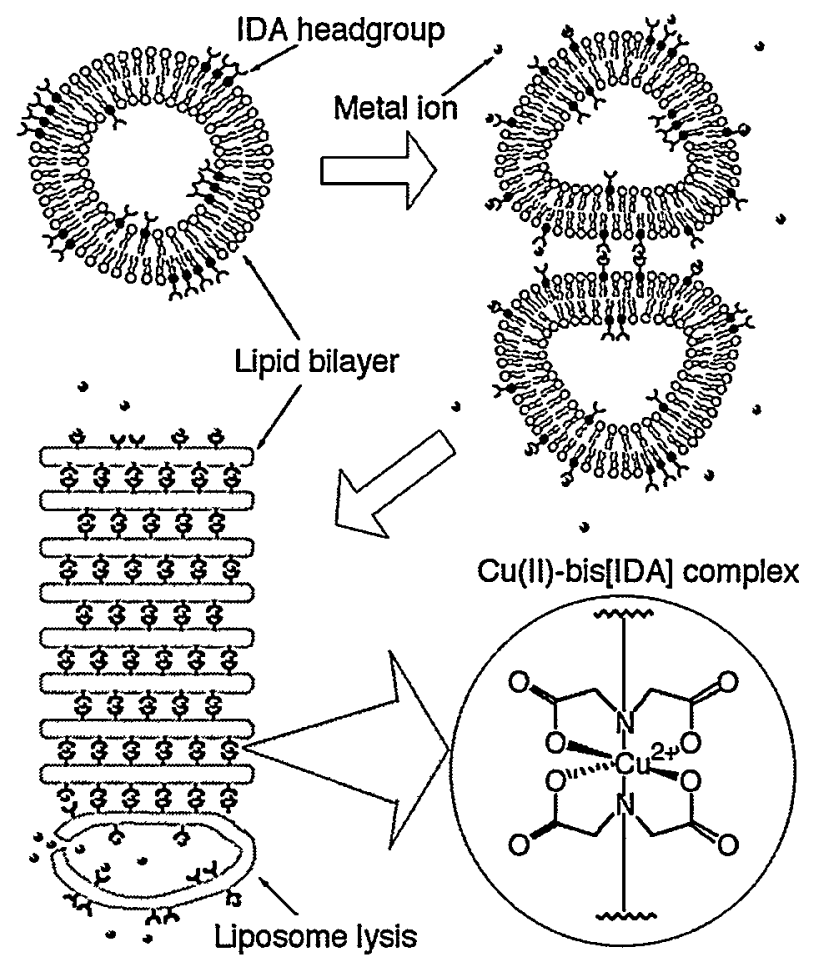

Figure 4. Proposed mechanism of lipid bilayer stack formation. Top left structure represents the initial liposome. Following metal ion addition, liposomes adhere to each other through $\mathrm{Cu}^{2+}$-bis[IDA] complexation (right) causing membrane flattening. Subsequent adhesion and liposome lysis leads to bilayer stacking (bottom left).

Investigations into the mechanism of formation of these bilayer stacks are currently underway. Such structurally defined, asymmetric materials may have potential use as scaffolding in nanoarchitecture or as structures with unique chemical or optical function.

Acknowledgement. Sandia is a multiprogram laboratory operated by Sandia Corporation, a Lockheed Martin Company, for the United States Department of Energy under Contract DE-AC04-94AL85000.

Supporting Information Available. Additional TEM images of lipid bilayer stacks. 


\section{Supporting Information}
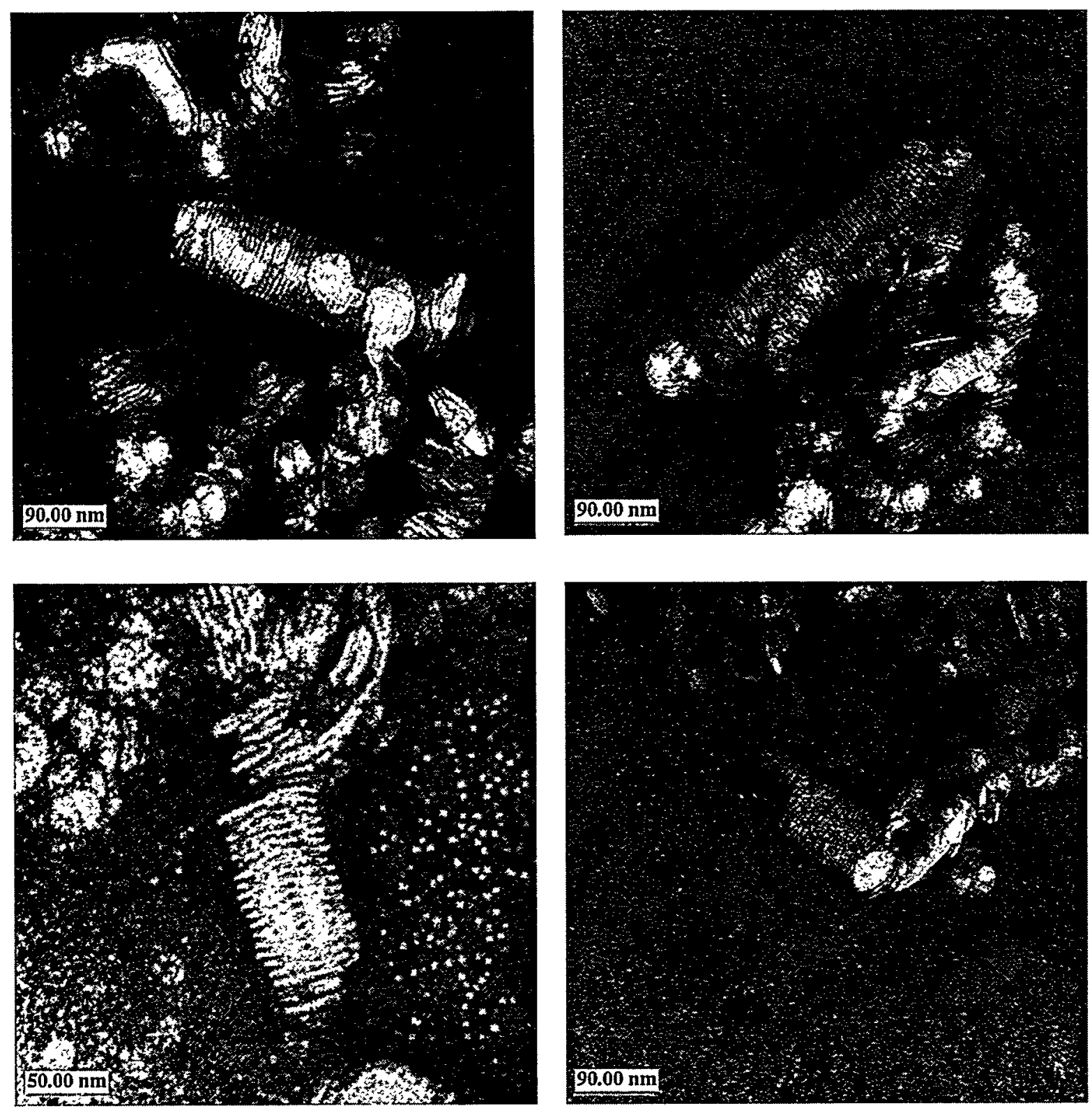

TEM images of columnar lipid bilayer stacks of 5\% PSIDA/DSPC (1.0 mM total lipid) composition formed through the introduction of $\mathrm{CuCl}_{2}(1 \mu \mathrm{M})$ followed by incubation at room temperature for 12 hours (ammonium molybdate stain). 